This item was submitted to Loughborough's Research Repository by the author.

Items in Figshare are protected by copyright, with all rights reserved, unless otherwise indicated.

\title{
Additive manufacturing: rapid prototyping comes of age
}

PLEASE CITE THE PUBLISHED VERSION

http://dx.doi.org/10.1108/13552541211231563

PUBLISHER

(c) Emerald Group Publishing Limited

VERSION

AM (Accepted Manuscript)

LICENCE

CC BY-NC-ND 4.0

REPOSITORY RECORD

Campbell, R.I., Dave Bourell, and lan Gibson. 2019. "Additive Manufacturing: Rapid Prototyping Comes of Age". figshare. https://hdl.handle.net/2134/11200. 
This item was submitted to Loughborough's Institutional Repository (https://dspace.lboro.ac.uk/) by the author and is made available under the following Creative Commons Licence conditions.

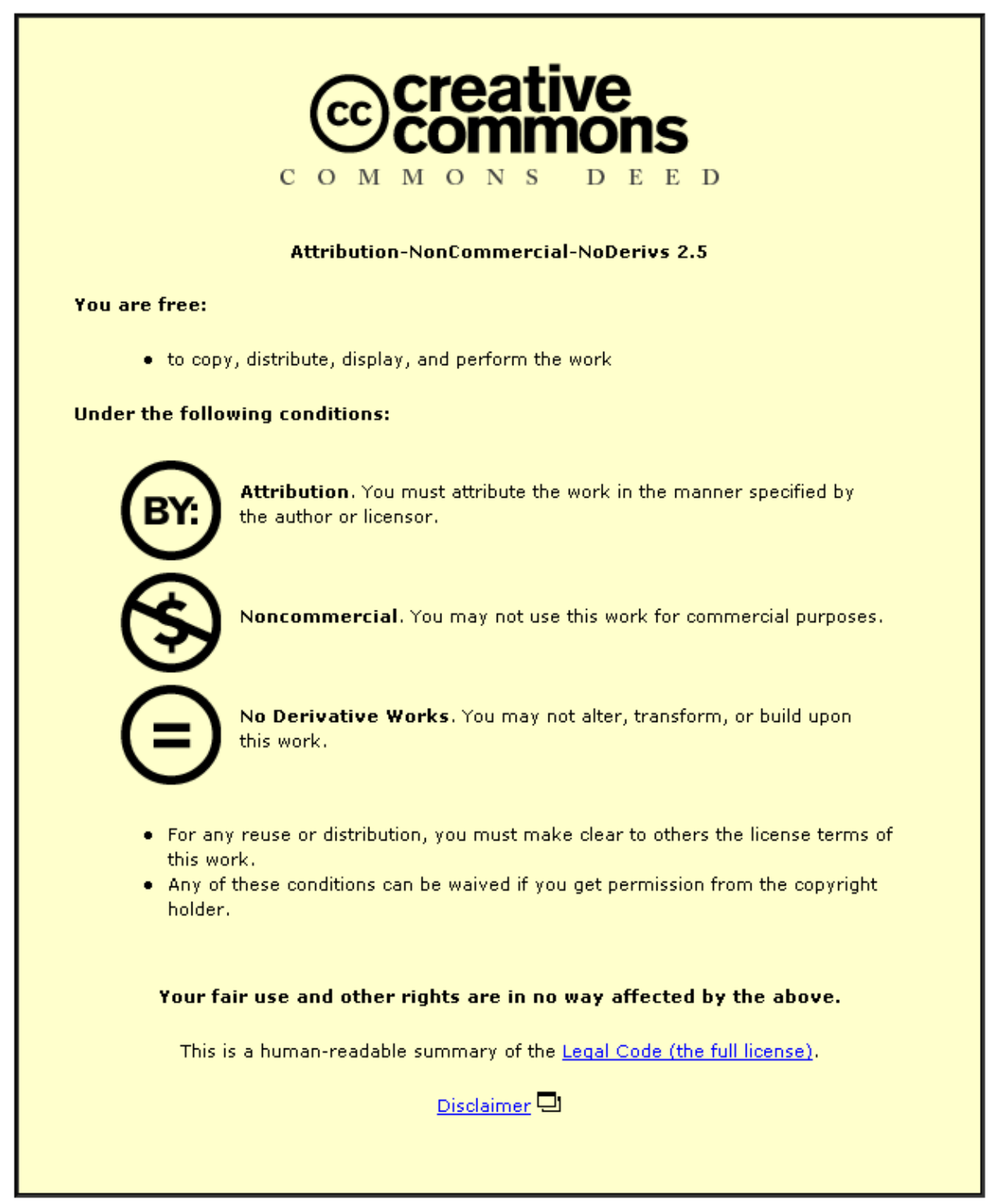

For the full text of this licence, please go to: http://creativecommons.org/licenses/by-nc-nd/2.5/ 


\title{
Additive Manufacturing: Rapid Prototyping comes of age
}

\author{
Ian Campbell, Dave Bourell, Ian Gibson
}

\begin{abstract}
This paper is a personalised view by the editors of the Rapid Prototyping Journal. It collects their years of experience in a series of observations and experiences that can be considered as a snapshot of where this technology is today. Furthermore, it attempts to predict how the future of Additive Manufacturing will look from the perspective of 3 key elements; applications, materials and design.
\end{abstract}

\section{Introduction}

Rapid Prototyping (RP) is a term that the manufacturing industry has struggled with for many years. Coined in the mid-1980s, it was used to describe a range of technologies that literally made prototypes of products in the early stages of development in a quick and automated manner. A variety of different techniques essentially combine planar layers of material, sequentially to form a 3-dimensional solid object. However, there is nothing in the term 'Rapid Prototyping' that alludes to this generic 'layer-based' approach. Furthermore, it is clear that current application of the technology goes far beyond merely prototyping.

Following the initial introduction of the key technologies, development of RP has been mostly incremental. The earliest machines included photopolymer curing (like Stereolithography, or SL from 3D Systems), powder sintering (like Selective Laser Sintering, or LS from DTM), filament extrusion (like Fused Deposition Modeling, or FDM from Stratasys), and sheet lamination (like Laminated Object Manufacturing, or LOM from Helisys). All of these technologies still exist today, with the exception of LOM, which is perhaps because it was the most material wasteful of these processes. Unlike other processes, in LOM a fixed amount of material per unit height is required to build a part regardless of the footprint or displacement volume of the part. This appears to have proven to be a somewhat restrictive approach. After some years, the inclusion of droplet-based deposition (including 3D wax printing by Solidscape, Polyjet from Objet and 3D Printing licensed through MIT) leveraged knowledge and expertise from a burgeoning printing industry to provide an option for potentially low-cost RP solutions.

Such activity has seen this technology, which was primarily aimed at producing prototyping models, develop to include tooling and direct manufacture of consumer products. All of the current commercial technologies have seen improvements in speed, part accuracy and material properties. Increasing interest and application from industry has subsequently led to reductions in machine and operating costs as well as an increase in the range of applications. Legitimisation of the industry is evidenced by the development of standards through ASTM and ISO. Further, recognition of the widening appeal of this technology has led to regular use of the terms Additive Manufacturing (AM, primarily within the manufacturing industry) and 3D Printing (by the general public at large). 
We therefore believe that it is an appropriate juncture in this development to discuss the current status of the technology and affected industries. The purpose of this paper is to consider the following key issues: -

- Industry and application trends: What industries are likely to use the technologies in the future and why?

- Material developments: What new materials are we likely to see in the future?

- Design implications: How are we going to be able to incorporate the technological advances that Additive Manufacturing can provide at the design stage?

We will approach each of these issues by way of explaining how they have been addressed in the past and currently, which should allow us to explain how they can impact the future development of Additive Manufacturing.

\section{Additive Manufacturing Industry and Applications}

Fundamentally, AM technology has hardly changed in recent years. The most recent 'new' commercial technology is probably the Objet Polyjet process, which deposits photopolymers using droplet deposition printheads (is this correct, would not the InVision machine from 3D Systems have predated the Objet systems?). Having said that, there have been dramatic improvements in existing technology that justifies the transition from RP to AM. As mentioned earlier, this transition is a culmination of a number of evolutionary developments in materials and processes combined with the reduction in costs of the technology that has served to open it up to a wider user base. However, there has also been a change in the mindset of users that has opened their eyes to new applications or even as a more effective replacement for existing applications.

Development of AM technology over the years has been exemplified by three key industries for differing reasons: -

Automotive manufacturers exploited the technology because of the ability to help new products get to the market quickly and in a predictable manner. Small savings in time and development costs can result in significant overall savings in vehicle development. An example approach is the use of AM as part of a bridge-tooling process to make parts available before the full production tool is ready. Manufacturers of high-end, low-volume automobiles are even using AM as a preferred production process for some parts because it is the most cost-effective approach to use. Of course, if all automotive manufacturers are (like now) using the same technologies then these competitive advantages will have been lost. However, it is obvious that AM is now a core technology for automotive product development.

Aerospace companies are interested in these technologies because of the ability to realise highly complex and high performance products. Integrating mechanical functionality, eliminating assembly features and making it possible to create internal functionality (like cooling channels, internal honeycomb style structures, etc.) combine to create lightweight structures with high geometric and functional complexity. Whilst there is already a flourishing industry for making polymer parts on high-performance military aircraft, 
probably the greatest interest in this industry is for the direct metal fabrication systems. It is relatively easy for direct-metal AM systems to process titanium, which makes it ideal for incorporation into carbon composite aircraft designs.

Medical industries are particularly interested in AM technology because of the ease in which 3D medical imaging data can be converted into solid objects. In this way devices can be customised to suit the needs of an individual patient. The industry has not taken off quite as much as might have been expected however, and this is probably due to the fact that it is driven bottom-upwards from the clinicians, which makes it difficult to establish a cost model. Where there has been opportunity for large-scale customisation (like with the Invisalign process and the in-the-ear hearing aids) then there has been good reward. Future development of this industry will progress slowly because of the need to ensure safety through organisations like the United States Food and Drug Administration (FDA).

The major limitations to AM are speed, accuracy, nonlinearity (different resolutions for XYZ axes, wall thickness), material properties and system cost. All of these are being addressed by the machine vendors. Materials will be dealt with specifically in the next section but a key development for direct manufacturing of parts has been the incorporation of additives to polymer matrix material to enhance the mechanical properties of resulting parts. Composites are therefore almost entirely used for direct manufacture or to enable manufacture through processes like short-run tooling.

System cost is a very subjective topic. Machines sell at a particular price partly because of their component and assembly costs but mostly because of their perceived value to users. If the manufacturing costs can be reduced, or the number of potential users becomes larger, then system price can be reduced. Recently, there has been a number of low-cost systems introduced to the market. These are aimed at entry level users and mainly for personal use and thus for comparatively high-volume sales. Machines are mainly based on the FDM technology since the relevant designs are generally available and the process is the easiest to synthesise. However, we can expect dropletbased technology to become widely available in the years to come when large-scale printer manufacturers decide the market is sufficient to accommodate the capital costs of manufacture.

Increased use of AM technology will inevitably mean it will find its way into more and more application areas. Some of these areas (including automotive, aerospace and medical) are very performance or safety critical, which requires careful process control and tracking to ensure high quality. Acceptance is going to be much easier if suitable standards are in place. Recent implementation of the ASTM and other international standards will certainly assist this acceptance process.

So what is the future for AM technology, beyond the incremental developments already discussed, that will influence new manufacturing applications? There follows a few possible predictions: -

If fabrication speed is significantly increased, parts will become available in minutes rather than hours. Consumers would be prepared to wait this short time for their parts to be made over the counter. Machines are likely to be seen in shopping malls and other locations where consumer parts can be made to order. Of course materials and the design process should also be enhanced to support such applications. 
It should be realised that AM is a process that can also be used in conjunction with other manufacturing processes that can also be automated. Future manufacturing equipments are likely to be designed to incorporate AM. Aerospace manufacturing is probably the first that will take advantage of this due to the obvious performance improvements that can be gained. These hybrid additive/subtractive technologies will not be as versatile as the existing AM machines; more likely specifically designed for a particular size and type of product, like turbine blades or wing struts. This approach is likely to be expensive but should achieve higher throughput for complex designs.

A number of AM systems have already demonstrated multiple material capability and the ASTM next generation AMF software standard has numerous features aimed at describing multiple material constructs. We are likely to see many more machines exploiting this capability in the future since this is not only a relatively easy thing to achieve with a number of the technologies, it also will result in interesting products that may have been extremely difficult to manufacture using conventional technology.

A completely new application area that is likely to develop is tissue engineering, where AM approaches are used to generate structures that will result in medical implants. These implants will contain cells that will eventually combine to form human tissue inside the body. AM is a key to achieving this but must also combine with new biochemical approaches for the ultimate goal. Already there a number of experimental systems available for researchers to explore the huge number of different options available.

\section{Material Development}

Materials have from the beginning of the technology been an important driving force for development. Like traditional manufacturing processes, the initial choice of material was tied to the constraints of the process. Photosensitive epoxy resins were used for stereolithography, powdered crystalline and semicrystalline polymers for laser sintering, adhesive paper for laminated object manufacturing, low-viscosity binders for inkjet printing and thermoplastics for fused deposition modelling. Advances in materials over the last 25 years fall generally into four categories.

The first has been the improvement in the intrinsic properties of AM parts engendered through improvements in the base materials. With increased demand came a fiscal motivation to tailor and optimise materials for best results. Examples are new formulations of SL resins, development of polyamides specifically for LS, and the roll-out of additional FDM filament materials. New materials were developed and marketed. Outstanding among this group are applications of a wide array of metallic materials and advanced engineering polymers such as EOS GmbH's PAEK and PEEK. As materials development efforts matured, composite approaches were undertaken to continue to improve properties whilst maintaining material characteristics necessary for successful AM processing. An example is mixing ceramic or metallic powder into SL resins or FDM filaments. In LS, powder has been alloyed with fine particulates to increase strength and improve electrical conductivity and flame retardant properties which are important for aerospace applications. 
The second advance was the application development of AM parts in the early 1990s in so-called "conversion technologies" in which the AM part geometry was maintained whilst a different material was substituted for the as-processed material. For virtually all processes, low-ash waxes and polymers were developed which could be used as patterns for subsequent lost-wax styled casting. The ability to easily produce porous ceramic parts was also exploited in the manufacture of casting cores. A notable example was Soligen whose early market niche focussed on ceramic cores produced by MIT's 3D printing process.

The third advance was in the area of post processing. As AM moved into direct competition with traditional manufacturing for service parts, the need arose to match service properties. For some systems, post processing was straightforward and comparable to traditional manufacturing. An example would be standard heat treatment of SLM/EBM titanium parts to remove martensitic structures. Another would be post processing of metallic AM parts to remove residual porosity by conventional sintering or hot isostatic pressing. More complex post-processing methods were developed specifically for all the commercial AM processes. For "indirect" AM processes, a transient binder was mixed with a non-polymeric matrix phase. The binder allows the mixture to be successfully processed into a shape using AM, but in post processing, the binder was transformed or removed ether thermally or chemically, and the resulting porous structure was densified by sintering or infiltration. An early example was 3D Systems' Laserform $^{\circledR}$ tool steel which was infiltrated with a copper-based alloy in a post-processing step after burning off the transient binder.

Lastly, third parties have entered the marketplace with novel developments in materials with concomitant improvements in service properties. These independent companies had the vision to see a market for AM feedstocks. Early developers were Ciba-Geigy (now Huntsman) and Somos for SL resins. More recent examples are CRP Technologies and Advanced Laser Materials whose product focus includes LS powder. Fundamental to the entry of third parties into AM is a commitment and philosophy that the materials offer advantages and benefits while being cost competitive.

So what does the future hold? One area where materials advances are foreseen to occur will be by do-it-yourselfers. There has in the last year or two been an explosion of relatively inexpensive AM devices based principally on FDM technology. Pioneered by Fab@Home developed at Cornell University and the RepRap project developed at the University of Bath, almost anything goes so long as it can be made to flow through a nozzle and harden. In contrast to this broad, often non-technical group of developers will be new and continued incremental advances by major AM machine developers and materials providers. The markets for AM have grown to a stage now that the technology is getting the attention of major feedstock developers and suppliers. It is anticipated that once these major producers are convinced that the time is right, one would expect to see new materials with improved manufacturability and service properties to enter the market. Increased competition will drive down costs as well which will be welcomed by the service bureaus and parts providers as well as consumers. One would hope that medium cost AM equipment will be developed in the next ten years as the primary patents expire, and that these devices, made available to the public on at least a neighbourhood or city-wide basis, would foster increased feedstock demand which in turn would accelerate entry of major suppliers into the marketplace with their new and improved materials.

\section{Design Implications}


The term "design", in regard to the use of AM, can cover several disciplines. These include industrial design, mechanical engineering design, architectural design and fashion design. AM is having an impact on all of these areas by enabling the manufacture of designs that were previously uneconomical or even impossible. Examples of these designs, first seen at research conferences or trade shows, are now finding their way into the mainstream, particularly in the on-line consumer product arena. A visual analysis of most of these designs indicates that there are a relatively small number of reasons why using AM was deemed desirable. These reasons can be categorised as follows:

1. User-fit requirement - a desire to customise the product to suit individuals' or groups of individuals' ergonomic requirements

2. Improved functionality - a desire to improve the product performance through the adoption of complex forms, both externally and internally

3. Parts consolidation - a desire to reduce the overall parts count in a product by producing a lower number of (usually) more complicated parts

4. Aesthetics - a desire to endow the product with specific design features that will increase its value to the customer

Those who are familiar with product design will recognise that a particular design feature would be capable of addressing more than one of these desires, e.g. a weight-saving functional structure may also produce a dramatic aesthetic form. This is of immense significance, as discussed later.

The implications upon the design process of using AM are twofold. Firstly, computer aided design software must be upgraded to handle the unique characteristics of parts designed for AM. For example, the ability to represent several materials or colours in the same model, the ability to have a gradual change from one material to another, the ability to assign a particular surface texture or pattern to a part and the ability to generate and represent complex internal structures. Many of these issues have been addressed by research and are beginning to find their way into commercial software. The newly released AMF format can handle many of these requirements and as such is poised to provide a replacement to the STL format which has difficulty in handling any of them.

The second implication relates to designers, namely, how will they be able to take advantage of all the opportunities that AM offers. Partly, this is a matter of education. Designers need to be made aware of the unique features of AM and encouraged to ignore the "design for manufacturing" limitations they have been used to. This can be done at university level through the embedding of AM into design course curricula. It can be achieved at a professional level through workshops and on-line training resources. However, there is also the matter of a need for increased creativity. It could be argued that the ultimate limitation to the shapes created by AM will be the imagination of the designer (or the programmers who create design automation software). Designers, of all disciplines, will need to unleash their creativity to come up with product, building and fashion designs that would previously have been implausible. In essence, how are we going to get designers to think what was unthinkable in the past?

Once again, education would seem to be key here, but not in terms of "what is learnt" but rather "how we teach". In the UK, certainly, students are divided into "arty types" and "scientific types" at 
quite a young age, typically 13 , when they have to choose their GCSE subjects. In other countries this divide happens later. Some students are given to believe that numbers and equations are not their strength and that they should concentrate on their creative flair. Others concentrate on science and technology and may even see studying art as a waste of time. No surprise then, when in later life they continue these thinking patterns throughout their careers. This poses a problem to designers who are expected to produce something both functional and aesthetically pleasing. Our educational system tends to produce designers who are capable of either one or the other. AM, on the other hand is uniquely capable of producing something which is both, without the need for compromise. We need "hybrid" designers who are capable of taking inspiration for their concepts from nature, fashion or the built environment and then converting these into product forms that will also perform efficiently and ergonomically. Such designers already exist but for AM to reach its full potential we need them to be the norm rather than the exception.

So what might the future of design for additive manufacturing be? If the both technical and human issues can be addressed, then a unique hybrid of the aesthetic and functional design disciplines might emerge. For example, going back to the dual purpose feature mentioned earlier, a single designer could perhaps take inspiration from nature (and calculations from software) to create visually stunning, weight-saving designs that will only be realisable using AM. Indeed, if the concept of beauty could be programmed into the software, then the hybrid designer might actually turn out to be a computer rather than a person!

\section{Conclusions}

We have discussed a range of Additive Manufacturing technologies, explaining how they came to be and their current status. Development of these technologies has progressed according to application, materials and how the designers have applied their creativity to such a unique manufacturing tools. Further we have discussed how recent developments have shaped the possible future for such technology. Whether we are accurate in our predictions, only time will tell. 